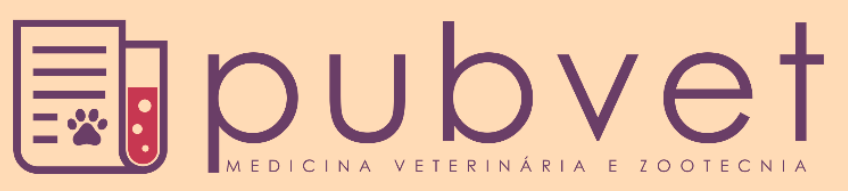

https://doi.org/10.31533/pubvet.v14n12a718.1-6

\title{
Deslocamento de abomaso à direita: Relato de caso
}

\author{
Andressa Juliana Fuelber ${ }^{1} \bullet$, Patricia Laura Funkler ${ }^{1}$, Tais Martinelli ${ }^{1} \bullet$, Vanessa Nadal ${ }^{\text {1* }}$ \\ ${ }^{I}$ Discentes do curso de Medicina Veterinária da Unochapecó/Chapecó- SC, Brasil \\ *Autor para correspondência, nadal.vanessa@unochapeco.edu.br
}

Resumo. A atividade leiteira vem se desenvolvendo constantemente e cada vez mais os produtores buscam melhores resultados produtivos, porém a seleção genética e o manejo nutricional que são adotados tornam as vacas mais suscetíveis a desenvolver doenças. $\mathrm{O}$ deslocamento de abomaso é a patologia mais frequente do trato gastrointestinal dos ruminantes, representando a principal causa de cirurgia abdominal em vacas de leite de alta produção. As principais causas estão relacionadas com o manejo alimentar na fase de transição, onde ocorre o fornecimento de dieta rica em energia, altamente fermentável e deficiente em fibras, entre outros. Os sinais clínicos incluem perda de apetite, queda acentuada na produção de leite, diarreia de coloração enegrecida, apatia, desidratação, timpanismo ruminal com motilidade ausente ou diminuída, som de líquido ao balotamento do flanco direito, som metálico à percussão, podendo inclusive, nos casos mais severos, virem a óbito. O diagnóstico é firmado através da anamnese somada ao exame clínico detalhado, que permite a auscultação de som metálico-timpânico no abomaso denominado "ping". Os tratamentos descritos na literatura são variados e existem métodos conservativos e cirúrgicos. A escolha da técnica depende da condição financeira do produtor e/ou do valor econômico da vaca, entretanto a intervenção cirúrgica faz-se necessária na maioria dos casos. Sendo assim, o presente artigo tem por objetivo descrever um caso de deslocamento de abomaso à direita em vaca leiteira de alta produção com histórico de aborto recente. Optou-se pelo tratamento mediante a técnica cirúrgica de abomasopexia com laparotomia pelo flanco direito com o animal em estação.

Palavras-chave: Abomasopexia, abomaso, diagnóstico

\section{Right abomasum shift: Case report}

Abstract. Dairy activity is constantly developing and more and more producers are looking for better productive results, however, the genetic selection and nutritional management that are adopted make cows more susceptible to developing diseases. Abomasum shift is the most frequent pathology of the gastrointestinal tract of ruminants, representing the main cause of abdominal surgery in high-producing milk cows. The main causes are related to food management in the transition phase, where the supply of a diet rich in energy, highly fermentable, and deficient in fiber occurs, among others. Clinical signs include loss of appetite, sharp drop in milk production, blackish-colored diarrhea, apathy, dehydration, ruminal tympanism with absent or diminished motility, the sound of liquid at the right flank, metallic sound at percussion, and more severe cases, die. The diagnosis is made through anamnesis in addition to a detailed clinical examination, which allows the auscultation of a metallic-tympanic sound in the abomasum called "ping". The treatments described in the literature are varied and there are conservative and surgical methods. The choice of technique depends on the financial condition of the producer and / or the economic value of the cow, however, surgical intervention is necessary in most cases. Therefore, the present article aims to describe a case of displacement of the right abomasum in a high-yielding dairy cow with a recent history of abortion. Treatment was chosen using 
the surgical technique of abomasopexy with laparotomy on the right flank with the animal in station.

Keywords: Abomasopexy, abomasum, diagnosis

\section{Desplazamiento del abomaso a la derecha: Reporte de caso}

Resumen. La actividad lechera está en constante desarrollo y cada vez más productores buscan mejores resultados productivos, sin embargo, la selección genética y el manejo nutricional que se adoptan hacen que las vacas sean más susceptibles a desarrollar enfermedades. El desplazamiento de abomaso es la patología más frecuente del tracto gastrointestinal de los rumiantes, representando la principal causa de cirugía abdominal en vacas lecheras de alta producción. Las principales causas están relacionadas con el manejo de los alimentos en la fase de transición, donde se produce el aporte de una dieta rica en energía, altamente fermentable y deficiente en fibra, entre otras. Los signos clínicos incluyen pérdida de apetito, disminución brusca de la producción de leche, diarrea de color negruzco, apatía, deshidratación, timpanismo ruminal con motilidad ausente o disminuida, sonido de líquido en el flanco derecho, sonido metálico a la percusión y en casos más graves, muere. El diagnóstico se realiza mediante anamnesis además de un examen clínico detallado, que permite la auscultación de un sonido metálico-timpánico en el abomaso denominado "ping". Los tratamientos descritos en la literatura son variados y existen métodos conservadores y quirúrgicos. La elección de la técnica depende de la situación económica del productor y / o del valor económico de la vaca, sin embargo, la intervención quirúrgica es necesaria en la mayoría de los casos. Por tanto, el presente artículo tiene como objetivo describir un caso de desplazamiento del abomaso derecho en una vaca lechera de alto rendimiento con antecedente reciente de aborto. El tratamiento se eligió mediante la técnica quirúrgica de abomasopexia con laparotomía en flanco derecho con el animal en posición.

Palabras clave: abomasopexia, abomaso, diagnóstico

\section{Introdução}

A seleção genética e o manejo nutricional utilizado com o intuito de melhorar a produção leiteira, tornam as vacas mais suscetíveis ao desenvolvimento de doenças. O deslocamento de abomaso vem sendo o distúrbio abomasal mais frequente e a principal causa de cirurgia em bovinos leiteiros (Patelli, 2014; Santarosa, 2010). Trata-se de uma doença multifatorial que acomete principalmente vacas leiteiras de alta produção no período pós-parto. O deslocamento de abomaso (DA) pode ocorrer à direta (DAD) ou esquerda (DAE), sendo o primeiro mais frequente. É bastante relacionado com o manejo alimentar e ocorre em animais que manifestam outras doenças, como hipocalcemia, cetose e retenção de placenta. Neste tipo de DA a víscera se desloca totalmente para o lado direito da cavidade abdominal, e em situações maiores de risco pode evoluir para o vólvulo abomasal (VA). No DAE o órgão vai migrar de sua posição anatômica normal, no assoalho do abdômen, para uma posição entre o rúmen e a parede abdominal esquerda (Gordo, 2009; $\underline{\text { Santos et al., 2009). }}$.

Dietas com altos níveis de concentrado para bovinos leiteiros resultam em hipomotilidade e atonia ruminal causando um acúmulo de gás abomasal (Câmara et al., 2011; Gordo, 2009; Santos et al., 2009). Demais fatores como desordens neuronais, estresse, estágio da gestação, doenças metabólicas e infecciosas, raça, idade e outros fatores genéticos, também são capazes de aumentar o risco de DA (Patelli, 2014; Santarosa, 2010).

A cetose diagnosticada antes do DA pode ser um fator que também esteja envolvido na ocorrência, uma vez que em quadros de cetose ocorre uma redução no consumo de matéria seca o que reduz o preenchimento ruminal, e com isso redução na motilidade abomasal. Sugere-se que o estágio avançado de prenhez, com um volume uterino aumentado ocupe uma porção do espaço do rúmen e, logo após o parto, com a saída do terneiro e fluídos existe uma predisposição anatômica para o abomaso deslocarse. Manter um volume ruminal normal é uma ferramenta importante para prevenir o deslocamento de abomaso (Câmara et al., 2011; Gordo, 2009; $\underline{\text { Santos et al., 2009). }}$ ). 
A sintomatologia clínica mais observada em animais com DAD consiste em uma anorexia total ou moderada, hipogalactia, defecação diminuída, com fezes enegrecidas e fétidas, diminuição da motilidade ruminal, em alguns casos pode haver ausência de ruminação, dor, cetonúria, odor bem característico de cetona na respiração, e abdômen bem afundado na fossa paralombar direita (Câmara et al., 2011; Gordo, 2009; Santos et al., 2009).

A doença é responsável por gerar perdas econômicas nos rebanhos leiteiros devido aos custos com tratamentos, diminuição da produção, leite descartado, aumento do intervalo entre partos, perda de peso corporal, descarte de animais e morte (Patelli, 2014; Santarosa, 2010).

O diagnóstico se dá pela ausculta e percussão do som timpânico (ping metálico) na região da fossa paralombar direita. $\mathrm{O}$ tratamento cirúrgico é o mais recomendado, tanto pela técnica de Abomasopexia Paramediana Ventral ou Abomasopexia pelo Flanco Direito (Patelli, 2014; Santarosa, 2010). Deve-se proceder a correção hidroeletrolítica do animal e tratar as doenças concomitantes.

Por se tratar de uma doença multifatorial, a prevenção deve ser feita pela identificação dos possíveis fatores predisponentes. $\mathrm{O}$ fator mais importante é o manejo nutricional do rebanho, evitar animais obesos nos estágios finais da gestação garantindo um manejo efetivo nesse período, garantir fontes de fibras efetivas para o rúmen para que ele possa estar sempre repleto. No final da gestação a dieta deve conter no mínimo $17 \%$ de fibra bruta, evitando a ocorrência de uma acidose ruminal pelo incremento de grãos neste período. As doenças que ocorrem após o parto como metrite, mastite, retenção de placenta que são viáveis para causar hipocalcemia precisam ser solucionadas (Câmara et al., 2011; Gordo, 2009; Hendrickson, 2010; Santos et al., 2009).

O presente artigo tem por objetivo descrever um caso de deslocamento de abomaso à direita em vaca leiteira de alta produção com histórico de aborto recente.

\section{Materiais e métodos}

Foi atendido na propriedade Nadal, no município de Planalto Alegre - SC, um animal da espécie bovina, fêmea, holandesa, preta e branca, com seis anos de idade. Peso corporal de $680 \mathrm{~kg}$, média de produção diária $40 \mathrm{~kg}$ leite, dieta de silagem de milho (20 kg/dia) e piquete de grama tifton (Cynodon dactilon). A propriedade vem trabalhando com sistema intensivo do tipo freestall.

O proprietário relatou um aborto de sete meses de gestação há 15 dias. Em um primeiro momento o animal estava sendo tratado para a expulsão dos restos fetais com Cloprostenol sódico $(3 \mathrm{ml} / \mathrm{dia})$ e Cipionato de estradiol (5 ml/dia). Para a infecção uterina foi utilizado por 7 dias Ceftiofur $(1 \mathrm{mg} / \mathrm{kg}$ em aplicação por via intramuscular).

Após três dias o animal apresentou sinal de apatia e emagrecimento progressivo. Entretanto, como o animal não reagia aos tratamentos, foi buscado auxílio de um Médico Veterinário. Ao exame clínico observou-se abaulamento da região ventral direita, fezes reduzidas de volume e amolecidas, arqueamento dorsal das costelas, com sensibilidade a palpação do abomaso e desidratação branda (5\%). $\mathrm{Na}$ auscultação observaram-se movimentos ruminais diminuídos e sons claros metálicos e agudos provenientes do abomaso. Os ruídos timpânicos foram melhores auscultados por meio da percussão com a ponta dos dedos na parede abdominal ao redor da área central do estetoscópio. Temperatura corporal, frequências cardíaca e respiratória encontravam-se dentro dos padrões fisiológicos para a espécie. A avaliação clínica definiu o diagnóstico em Deslocamento de Abomaso à Direita.

Desta forma, optou-se pelo tratamento mediante a técnica cirúrgica de abomasopexia com laparotomia pelo flanco direito com o animal em estação. Como protocolo sedativo utilizou-se $2,00 \mathrm{~g} / \mathrm{kg}$ de cloridrato de lidocaína (anestésico local sem vasoconstritor) por via intradérmica e bloqueio locoregional em L invertido. Procedeu-se a antissepsia com água e sabão com posterior tricotomia e lavagem com água e cloreto de benzalcônio ((15 g) bactericida, fungicida e viricida). Na fossa paralombar direita foi realizada uma incisão dorsoventral, com cerca de $25 \mathrm{~cm}$. Após incidir a pele, o músculo oblíquo abdominal externo, oblíquo abdominal interno, transverso do abdome e peritônio, inspecionou-se a cavidade abdominal e foi possível observar a presença do abomaso em posição anormal deslocado na região dorsal direita. Desta forma, fez-se uma sutura contínua, utilizando fio de sutura Nylon, na 
curvatura maior do abomaso, deixando dois segmentos de fio com cerca de 1 metro de comprimento para posterior fixação no abdômen ventral direito.

Realizou-se a descompressão do abomaso, utilizando agulha e, então procedeu-se o reposicionamento do abomaso na respectiva área anatômica. Para esse procedimento, utilizou-se agulha curva, iniciando com a extremidade cranial do fio. Protegendo a agulha com a mão, introduziu-se seguindo a parede lateral, levando o abomaso em sua posição normal. Atravessou-se a agulha na parede lateral ventral direita. Fez-se o mesmo procedimento com a extremidade caudal do fio, desta forma, permitiu-se o reposicionamento do abomaso em sua posição anatômica fisiológica.
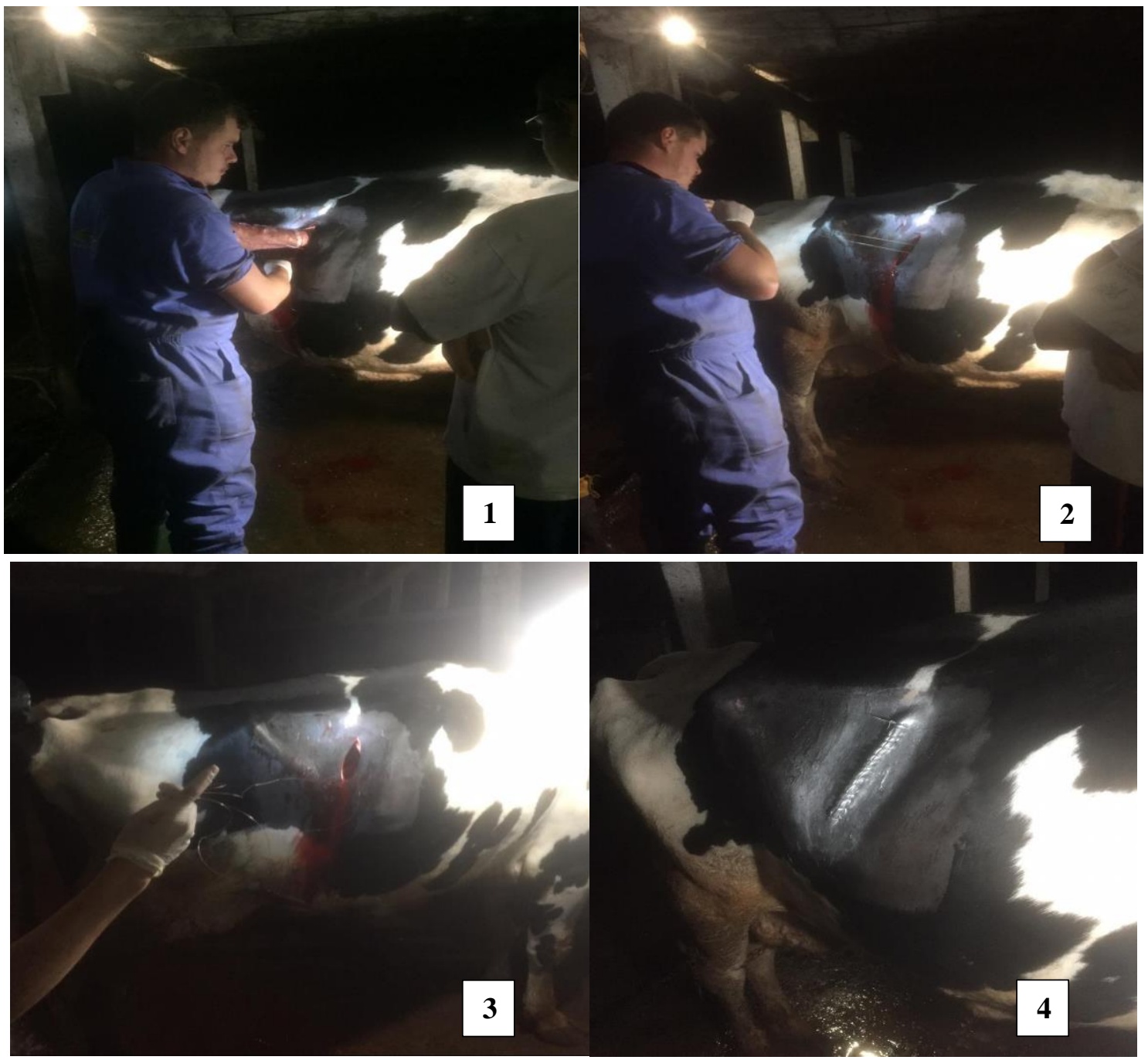

Foto 1 e 2. Pontos no abomaso para fazer reposicionamento. Foto 3 e 4. Procedimento da cirurgia de deslocamento de abomaso à direita. Sutura das camadas e finalização

Colocou-se uma tampa de borracha de medicamento na ponta de cada fio e posterior nó de cirurgião com mais três sobre nós em cada fio. A sutura na fossa paralombar direita foi realizada em camadas, onde o peritônio foi ligado ao músculo transverso do abdome com fio de Nylon em padrão de sutura contínuo festonado. A segunda camada foi pelo padrão simples contínuo, aproximando o músculo oblíquo abdominal externo ao músculo oblíquo abdominal interno. Em sequência realizou-se a sutura de pele através do padrão de sutura com pontos contínuos simples. Antes do fechamento da sutura de pele foi adicionada na sutura uma seringa de Vetmast plus V.L (pomada oleosa composta por antibiótico, antifúngico e anti-inflamatório). Na sutura foi utilizado spray repelente diariamente. Realizou-se também administração de glicose, cálcio e associação de vitaminas e aminoácidos, por via intravenosa, seguido de antibióticoterapia com sulfadoxina $(20 \mathrm{~g} / \mathrm{kg})$ e trimetropina $(4 \mathrm{~g} / \mathrm{kg})$ por via intramuscular, a cada 24 horas por dois dias. Realizou-se a retirada dos pontos de pele e a alta clínica do animal 14 dias após o procedimento cirúrgico. 


\section{Discussão}

A vaca deste relato teve um aborto recente, desta forma relaciona-se com o relato de Câmara et al., (2011); Gordo (2009); Hendrickson (2010) e Santos et al. (2009), onde a maior frequência dos casos de DAD ocorre no pós-parto, com 50-70\% dos casos durante o primeiro mês, enquanto aproximadamente $20 \%$ das vacas não possuem correlação com o puerpério ou altas produções leiteiras. Segundo Santarosa (2010), bovinos que apresentam DA sem complicações tem um histórico de hiporexia ou anorexia, redução na produção fecal, redução na frequência e intensidade das contrações ruminais além de queda significativa na produção e perda de peso. A temperatura, frequência cardíaca e respiratória permanecem dentro dos padrões físiológicos, exceto quando a uma compressão diafragmática, em casos de timpanismo crônico.

Animais com DAD durante a percussão/auscultação produzem uma área ampla de som metálico, podendo se estender do $8^{\circ}$ ao $9^{\circ}$ espaço intercostal até a fossa paralombar direita, em alguns casos ainda pode ser observado som líquido que está associado ao balotamento do flanco e diz que a o principal achado no exame clínico para a confirmação do diagnóstico é a associação entre a presença de som de líquido no balotamento do flanco direito constatação de estrutura similar a uma víscera distendida com formato de meia lua e área com som de chapinhar metálico no antímero correspondente ao lado do deslocamento (Patelli, 2014; Santarosa, 2010).

Nos resultados hematológicos, bovinos com DAD apresentam na maioria dos casos leucocitose por neutrofilia com desvio à esquerda degenerativo, associados a hiperfibrinogenemia, que são sugestivos de um processo inflamatório (Cardoso, 2008; Patelli, 2014; Santarosa, 2010).

Para iniciar o tratamento, é necessário levar em consideração o custo do tratamento, perdas econômicas previstas, prognóstico para retornar à produção, interesse do proprietário em tratar o animal. $\mathrm{O}$ procedimento cirúrgico é o mais indicado. Para Câmara et al. (2011) e Motta et al. (2015), as técnicas mais utilizadas são, em ordem decrescente, a omentopexia pelo flanco direito (OFD) e omento-abomasopexia, pelo flanco direito (OAFD), e a abomasopexia pelo flanco esquerdo (AFE); entretanto, a utilização de outras técnicas e a sua frequência de uso varia de acordo com a opção e afinidade de cada cirurgião.

Nos casos de vacas diagnosticadas com DAD, a intervenção deve ser iniciada o mais rápido possível, pelo fato de ocorrência de volvo abomasal secundária, o que é considerado grave, podendo levar o

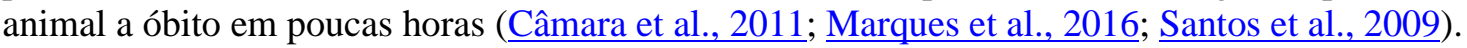

\section{Conclusão}

Este estudo relatou um caso de deslocamento de abomaso à direita em vaca leiteira considerada de alta produção, sendo corrigida com a técnica cirúrgica de abomasopexia com laparotomia pelo flanco direito. O DAD é uma enfermidade com caráter econômico importante para a produção leiteira sendo dependente da introdução de manejos que exigem grandes adaptações do animal. O prognóstico é considerado reservado em todos os casos, principalmente quando existe comprometimento grave do órgão ou alterações sistêmicas importantes. Tendo isso em vista conclui-se que a prevenção é a melhor maneira de evitar prejuízos na produção leiteira.

\section{Referências bibliográficas}

Câmara, A. C. L., Afonso, J. A. B., \& Borges, J. R. J. (2011). Métodos de tratamento do deslocamento de abomaso em bovinos. Acta Veterinaria Brasilica, 5(2), 119-128.

Cardoso, F. (2008). Indicadores hematológicos, bioquímicos e ruminais no diagnóstico do deslocamento de abomaso à esquerda em vacas leiteiras do Sul do Brasil. Pesquisa Agropecuária Brasileira, 43, 141-147.

Gordo, R. I. N. (2009). Contribuição para o estudo do deslocamento do abomaso numa exploração leiteira da região de Montemor-o-Velho. In Faculdade de Medicina Veterinária: Vol. Master of. Universidade Técnica de Lisboa.

Hendrickson, D. A. (2010). Técnicas cirúrgicas em grandes animais (Vol. 1, p. 238 p.). Guanabara Koogan.

Marques, T. O., Sá, M. A. F., Pereira, N. B. A., Galvão, A., \& Rocha, F. S. (2016). Deslocamento de abomaso à esquerda em bovinos de leite no sul de Minas Gerais - Relato de quatro casos. . Revista Científica de Medicina Veterinária, 27, 1-10. 
Motta, R. G., Motta, I. G., Botelho, A. C. F. A., Gheller, J. M., Souza, A. M., Baldin, T., Momo, R., Schreiber, J., Barbosa, L. C., \& Ribeiro, M. G. (2015). Deslocamento de abomaso à esquerda em bovino-relato de caso. Atas de Saúde Ambiental-ASA, 2(3), 53-61.

Patelli, E. M. (2014). Deslocamento do abomaso à esquerda em bovinos leiteiros: da etiologia ao diagnóstico. In Faculdade de Medicina e Veterinária: Vol. Graduação. Universidade Estadual de São Paulo.

Santarosa, B. P. (2010). Deslocamento de abomaso em vacas leiteiras. In Faculdade de Medicina Veterinária e Zootecnia: Vol. Graduação. Universidade do Estado de São Paulo.

Santos, P. R., Avanza, M. F. B., \& Pereira, D. M. (2009). Deslocamento do abomaso para o lado direito, na raça bovina de leite - relato de caso clínico. Revista Cientifica Eletrônica de Medicina Veterinária, $7(13), 1-5$.

Campbell, J. W., Harvey, T. G., McDonald, M. F., \& Sparksman, R. I. (1996). Transcervical insemination in sheep: an anatomical and histological evaluation. Theriogenology, 45(8), 15351544. DOI: https://doi.org/10.1016/0093-691X(96)00121-5

Hawk, H. W. (1983). Sperm survival and transport in the female reproductive tract. Journal of Dairy Science, 66(12), 2645-2660. DOI: https://doi.org/10.3168/jds.s0022-0302(83)82138-9

Kershaw, C. M., Khalid, M., McGowan, M. R., Ingram, K., Leethongdee, S., Wax, G., \& Scaramuzzi, R. J. (2005). The anatomy of the sheep cervix and its influence on the transcervical passage of an inseminating pipette into the uterine lumen. Theriogenology, 64(5), 1225-1235. DOI: https://doi.org/10.1016/j.theriogenology.2005.02.017

Khalil, K. E., Allai, L., Fatet, A., Benmoula, A., Hamidallah, N., Badi, A., Moussafir, Z., Ibnelbachyr, M., \& El Amiri, B. (2018). Morphometry and depth of inseminating catheter penetration in prolific and non-prolific ewes at different ages: A post mortem study. Animal Reproduction Science, 196, 43-47. DOI: https://doi.org/10.1016/j.anireprosci.2018.06.017

Maxwell, W. M. C., \& Salamon, S. (2000). Storage of ram semen. Animal Reproduction Science, 62, 77-111. DOI: https://doi.org/10.1016/S0378-4320(00)00155-X

Moura, D. S., Lourenço, T. T., Moscardini, M. M., Scott, C., Fonseca, P. O., \& Souza, F. F. (2011). Aspectos morfológicos da cérvice de ovelhas. Pesquisa Veterinária Brasileira, 31, 33-38. DOI: https://doi.org/10.1590/s0100-736x2011001300006

Sayre, B. L., \& Lewis, G. S. (1997). Fertility and ovum fertilization rate after laparoscopic or transcervical intrauterine artificial insemination of oxytocin-treated ewes. Theriogenology, 48(2), 267-275. DOI: https://doi.org/10.1016/s0093-691x(97)84074-5

Weems, C. W., Weems, Y. S., \& Randel, R. D. (2006). Prostaglandins and reproduction in female farm animals. The Veterinary Journal, 171(2), 206-228. DOI: https://doi.org/10.1016/j.tvjl.2004.11.014

Wulster-Radcliffe, M. C., \& Lewis, G. S. (2002). Development of a new transcervical artificial insemination method for sheep: effects of a new transcervical artificial insemination catheter and traversing the cervix on semen quality and fertility. Theriogenology, 58(7), 1361-1371. DOI: https://doi.org/10.1016/s0093-691x(02)01042-7

Wulster-Radcliffe, M. C., Wang, S., \& Lewis, G. S. (2004). Transcervical artificial insemination in sheep: effects of a new transcervical artificial insemination instrument and traversing the cervix on pregnancy and lambing rates. Theriogenology, 62(6), 990-1002. DOI: https://doi.org/10.1016/j.theriogenology.2003.12.031

Histórico do artigo:

Recebido: 1 de julho, 2020

Aprovado: 8 de agosto, 2020.

Disponível online: 6 de novembro, 2020.
Licenciamento: Este artigo é publicado na modalidade Acesso Aberto sob a licença Creative Commons Atribuição 4.0 (CC-BY 4.0), a qual permite uso irrestrito, distribuição, reprodução em qualquer meio, desde que o autor e a fonte sejam devidamente creditados. 